\title{
Efecto remediador de extractos de Sapindus saponaria sobre un suelo contaminado con gasoil a nivel de laboratorio
}

Remedial effect of Sapindus saponaria extracts on a soil contaminated with diesel at laboratory level

${ }^{1, a, c}$ Tomás Darío Marín-Velásquez

${ }^{1, b, d}$ Dany Day Josefina Arriojas-Tocuyo

\section{ARTÍCULO ORIGINAL \\ ${ }^{1}$ Universidad de Oriente, Escuela de Ingeniería y Ciencias Aplicadas Departamento de Ingeniería de Petróleo. Maturín, Monagas, Venezuela. \\ ${ }^{2}$ Gerencia de Manejo de datos, Petróleos de Venezuela. Punta de Mata, Monagas, Venezuela. \\ Correspondencia: \\ 'tmarin@protonmail.com \\ barriojasd@gmail.com}

ORCID: 0000-0002-3334-5895 ORCID: 0000-0002-8192-9641

Palabras clave: Árbol de jabón, hidrocarburos, impacto ambiental, remediación, Sapindus saponaria

Keywords: S o a p tree, hydrocarbons, environmental impact, remediation, Sapindus saponaria

\section{Información adicional}

Presentado: 07/01/2021

Aprobado: 02/05/2021

\section{RESUMEN}

Se evaluaron dos extractos de Sapindus saponaria como remediadores de un suelo contaminado con gasoil, a nivel de laboratorio. El suelo se contaminó con $100 \mathrm{ml}$ de gasoil por kilogramo y se trató con extractos acuosos e hidroalcohólico en dosis de 50, 100 y $150 \mathrm{ml}$ por kilogramo de suelo contaminado, con una muestra control por atenuación natural. El diseño fue completamente aleatorio con seis tratamientos, un bloque patrón, tres replicas por tratamiento y una variable respuesta (porcentaje de extraíbles con n-hexano, HEM), según norma EPA 9071b. Se tomaron muestras de cada unidad experimental a los 15, 30 y 45 días y se determinó el HEM. La prueba estadística fue ANDEVA factorial con criterios múltiples de Tukey y Dunnet y significancia $\alpha=0,05$ para establecer la influencia de los factores experimentales sobre la variable respuesta. Los extractos de $S$. saponaria tuvieron un efecto positivo, aumentando la eficiencia de remediación, respecto al control, de forma estadísticamente significativa, siendo el tratamiento con $150 \mathrm{ml}$ de extracto hidroalcohólico el más eficiente con una remoción de $93.9 \%$ del gasoil, aun cuando estadísticamente no hubo diferencia entre los extractos y los factores que influyeron fueron la cantidad de extracto y el tiempo de la prueba.

\section{ABSTRACT}

Two extracts of Sapindus saponaria were evaluated as remediators of a diesel contaminated soil, at laboratory level. The soil was contaminated with $100 \mathrm{ml}$ of gasoil per kilogram and was treated with aqueous and hydroalcoholic extracts in doses of 50,100 and $150 \mathrm{ml}$ per kilogram of contaminated soil, with a control sample by natural attenuation. The design was randomized blocks with six treatments, a standard block, three replicates per treatment and a variable response (percentage of extractables with n-hexane, HEM), according to EPA 9071b. Samples were taken from each experimental unit at 15,30 and 45 days and the HEM was determined. The statistical design was factorial ANOVA with multiple criteria of Student-NewmanKeuls and significance $\alpha=0.05$, to establish the influence of the experimental factors on the response variable. The extracts of $S$. saponaria had a positive effect, increasing the remediation efficiency, with respect to the control, in a statistically significant way, being the treatment with $150 \mathrm{ml}$ of hydroalcoholic extract the most efficient with a removal of $93.9 \%$ of the gasoil, even when statistically there was no difference between the extracts and the factors that influenced were the amount of extract and the time of the test. 


\section{INTRODUCCIÓN}

La contaminación de suelos producida por el petróleo y sus derivados es un problema al que se enfrenta la industria petrolera desde sus inicios. Los procesos que permiten la extracción, transporte y procesamiento del petróleo producen la contaminación del suelo debido a accidentes que generan derrames, que pueden ocurrir como consecuencia de filtraciones en tanques de almacenamiento, pozos, patios de almacenamiento y bombeo, y ruptura o daños de oleoductos y poliductos (Chan-Quijano et al., 2015). La presencia de los hidrocarburos componentes del petróleo pueden impedir el intercambio gaseoso del suelo con la atmósfera, lo que lleva a procesos físico-químicos, como la evaporación y penetración que, en función de parámetros como tipo de hidrocarburo, temperatura, humedad, textura del suelo y cantidad derramada, pueden ser procesos más o menos lentos, lo que ocasiona una mayor toxicidad (Benavides et al., 2006).

En los últimos años se ha prestado mucha atención a los métodos biológicos para la recuperación de suelos contaminados con hidrocarburos, siendo la biorremediación y la atenuación natural los métodos más utilizados (Corona-Ramírez \& Iturbe-Argüelles, 2005). En la naturaleza, se realizan procesos de atenuación natural en donde los hidrocarburos son eliminados del suelo por procesos que involucran acción microbiana (biodegradación) y fisicoquímicos como volatilización, percolación y drenaje superficial (Trujillo \& Ramírez, 2012). La biorremediación incluye dos procesos específicos cuya finalidad es acelerar el proceso de descontaminación del suelo, estos procesos son la bioaumentación o adición al suelo de microorganismos capaces de degradar los hidrocarburos y la bioestimulación, técnica que consiste en adicionar al suelo soluciones acuosas con nutrimentos que aumentan la actividad microbiana degradadora (Gómez et al., 2009). La biorremediación es una técnica que se ha aplicado exitosamente para remediar suelos contaminados con hidrocarburos (Rodríguez, 2017). La principal característica de las técnicas biológicas es su bajo costo, además de que se puede recuperar el suelo sin obtener elementos secundarios peligrosos. Sin embargo, la adaptabilidad de la población microbiana, así como los requerimientos de nutrientes, es lo que permite degradar a los contaminantes por medio de reacciones (Corona-Ramírez \& Iturbe-Argüelles, 2005).

El petróleo es una fuente importante de contaminación ambiental, debido a que está compuesto por una gran cantidad de hidrocarburos, desde parafinas ligeras hasta compuestos aromáticos policíclicos que son difíciles de degradar. La diversidad de hidrocarburos en el petróleo produce variaciones considerables en el color, el olor y demás propiedades que son función además del origen del petróleo (Speigh, 2002). De igual forma, el petróleo muestra variaciones en los tipos moleculares presentes en su composición, que incluyen compuestos heteroátomos como nitrógeno, oxígeno, azufre y metales. Se ha comprobado que los hidrocarburos son utilizados por los microorganismos del suelo como fuente de energía y de carbono (Laczi, et al., 2015; Rajasekar, 2016). Entre los derivados del petróleo, el gasoil se caracteriza por estar compuesto por una mezcla compleja de hidrocarburos parafínicos, nafténicos, olefínicos, aromáticos y poliaromáticos, los cuales poseen número de carbono entre $\mathrm{C}_{10} \mathrm{y}_{18}$, con un intervalo de ebullición entre 80 y $400{ }^{\circ} \mathrm{C}$ (BP Oil España, 2000; Reglamento Técnico Centroamericano, 2006). Al igual que el petróleo crudo, cuando el gasoil es liberado en el ambiente los componentes más ligeros tienden a evaporarse y a sufrir fotooxidación al reaccionar con los radicales hidroxilos. Lo anterior también les ocurre a los hidrocarburos más pesados, pero lo normal es que los mismos sean absorbidos por el suelo o los sedimentos. La mayoría de los componentes del gasoil son posibles de biodegradar bajo condiciones aeróbicas, es decir, en presencia de oxígeno (Repsol, 2016).

Al ser la biorremediación la técnica más utilizada para la recuperación de suelos contaminados con hidrocarburos, se han realizado muchas investigaciones donde se han aplicado la bioaumentación y la bioestimulación, demostrándose que esta última es la opción más adecuada por su bajo costo y su eficiencia, pues al utilizar las sepas de microorganismos autóctonos se asegura que los mismos se adapten mejor a la nueva condición generada por los hidrocarburos (Díaz-Martínez et al., 2013) siendo el uso de nutrientes químicos, la técnica más estudiada (Islas-García et al., 2016; Osoria, 2018). La remediación de suelos contaminados con gasoil ha sido estudiada por bioaumentación con microorganismos autóctonos, lográndose una reducción significativa de los hidrocarburos (Arrieta et al., 2012; Loroña et al., 2018). Así mismo, se ha demostrado que con el uso de productos comerciales y atenuación natural se logra una 
reducción significativa en la cantidad de hidrocarburos en un suelo que ha sido contaminado con gasoil (Romaniuk et al., 2007). También se han utilizado lodos residuales de una planta de tratamiento de agua para remediar un suelo contaminado con diésel, lográndose alcanzar los límites permisibles (Martínez-Prado et al., 2011).

El uso de extractos de origen vegetal también ha sido estudiado como medio acelerador del proceso de biorremediación de suelos contaminados con hidrocarburos, demostrándose su potencial. Entre ellos el extracto de la cáscara Citrus sinensis (Marín, 2017; Marín \& Arriojas, 2020; Marín-Velásquez \& BarrutiaBarreto, 2020) y el de la fibra de cáscara de Cocos nucifera (Marín et al., 2018) obteniéndose en ambos casos porcentajes de remediación superiores a la atenuación natural al aplicar los extractos. Tomando como referencia lo anterior, se planteó el estudio del uso de extractos del fruto de Sapindus saponaria, la cual es una planta de la familia Sapindaceae, que puede llegar a una altura de $12 \mathrm{~m}$ y se distribuye por toda América desde el sur de Estados Unidos, Centro América, Venezuela, Ecuador, Perú, Colombia y Brasil (Sánchez \& Silva, 2008). El fruto de $S$. saponaria produce una sustancia jabonosa, compuesta principalmente por un surfactante o jabón natural denominado saponina, por esta razón se le conoce también con el nombre común de jaboncillo, aunque en Venezuela se le llama "para para" (Alarcón, 2016; Whaley et al., 2009). Dado el potencial de la planta para producir sustancias que pueden ser utilizadas por diferentes industrias, y que los extractos obtenidos de sus frutos son biodegradables con potencial detergente y surfactante, se ha buscado otra fuente alternativa de extractos para la remediación de suelos contaminados con hidrocarburos, diferente a los utilizados comúnmente. Por lo tanto, se planteó la presente investigación, en la que se evalúa el efecto de dos extractos (acuoso e hidroalcohólico) del fruto de $S$. saponaria en la remediación de un suelo contaminado con petróleo a nivel de laboratorio.

\section{MATERIALES Y MÉTODOS}

\section{Muestra de suelo}

La muestra de suelo se obtuvo de un terreno ubicado en la población de El Furrial en Maturín, Estado Monagas, Venezuela, cuyas coordenadas son $9^{\circ} 41^{\prime} 08.72^{\prime \prime} \mathrm{N}$ y $63^{\circ} 26^{\prime} 31.07^{\prime} \mathrm{O}$. El muestreo realizado fue al azar simple y de forma superficial $(0-20 \mathrm{~cm}$ de profundidad). Se tomaron submuestras de todo el campo, para luego mezclarlas y obtener una muestra compuesta. En total se recolectaron 25 submuestras de $1 \mathrm{~kg}$ cada una, según procedimiento recomendado por Lizcano et al. (2017), obteniéndose una muestra compuesta de $25 \mathrm{~kg}$.

\section{Caracterización de la muestra de suelo}

La muestra compuesta de suelo se analizó, para obtener las propiedades relevantes para el estudio realizado (Tabla 1), siguiendo los procedimientos definidos por Bazán (2017).

\section{Tabla 1}

Propiedades medidas a la muestra compuesta de suelo

\begin{tabular}{llll}
\hline Propiedad & Método de medición & Unidad & Abreviatura \\
\hline Humedad & Pérdida de peso & $\%$ & $\mathrm{H}$ \\
Densidad Real & Método del picnómetro & $\mathrm{g} / \mathrm{ml}$ & $\mathrm{DR}$ \\
Textura & Método de Sedimentación & ----- & \\
pH & Método Potenciométrico & ---- & \\
Materia Orgánica & Método Walkley y Black & $\%$ & $\mathrm{MO}$ \\
Carbono Orgánico & Método Walkley y Black & $\%$ & $\mathrm{CO}$ \\
Extraíbles con hexano & Método EPA 9071b & $\%$ & $\mathrm{HEM}$ \\
\hline
\end{tabular}




\section{Muestra de gasoil}

La muestra de gasoil utilizada fue adquirida de un surtidor de combustible comercial. Se utilizó gasoil de tipo DB2, cuyas características estandarizadas se muestran en la Tabla 2.

Tabla 2

Propiedades del combustible gasoil

\begin{tabular}{lllll}
\hline Propiedad & Método de medición & Valor & Unidad & Abreviatura \\
\hline Gravedad API & ASTM D1298 & 34 & Grados & API \\
Viscosidad $\left(40{ }^{\circ} \mathrm{C}\right)$ & ASTM D445 & Máx. 4,1 & $\mathrm{cSt}$ & $\mu$ \\
Nro. de Cetanos & ASTM D613 & 50 & -- & NC \\
Punto de Inflamación & ASTM D93 & Mín. 52 & ${ }^{\circ} \mathrm{C}$ & PIF \\
Agua y Sedimentos & ASTM D1796 & 0,05 & $\%$ & AyS \\
Azufre & ASTM D4294 & 0,05 & $\%$ & AZ \\
\hline
\end{tabular}

\section{Extractos de S. saponaria}

Se obtuvieron los frutos maduros de árboles de $S$. saponaria, ubicados en las localidades de Potrerito en el Norte del Estado Monagas, Venezuela, cuyas coordenadas son 942'13.09” N y 6329'44.25" O. La obtención de los extractos se realizó utilizando el método de extracción sólido-líquido continuo de Soxhlet durante cuatro horas, mediante una batería de cuatro extractores con capacidad de $500 \mathrm{ml}$ cada uno. En dos extractores se obtuvo extracto utilizando agua como extractor y en los dos restantes el extractor fue alcohol etílico al 70\%. Se utilizó en cada extractor $100 \mathrm{~g}$ de fruto. En total se obtuvo un litro de cada extracto y se almacenaron en botellas de vidrio ámbar para ser utilizados luego en los ensayos.

\section{Diseño experimental}

Se tomaron $23 \mathrm{~kg}$ de suelo y se mezclaron con el gasoil en una proporción de $100 \mathrm{ml}$ por kg de suelo (Arrieta et al., 2012; Marín, 2017). El suelo contaminado se dejó en reposo por siete días y se caracterizó de acuerdo a los métodos establecidos en la Tabla 1 . Se establecieron 18 unidades experimentales de $1 \mathrm{~kg}$ cada una, a las que se le aplicaron seis tratamientos, tres con el Extracto Acuoso (EA) y tres con el Extracto Hidroalcohólico (EH), de acuerdo a lo que se muestra en la Tabla 3. Además, se dejaron tres unidades experimentales adicionales como control (MC), a las que no se le aplicó ningún tratamiento, para ser analizadas por atenuación natural.

Tabla 3

Descripción de los tratamientos aplicados

\begin{tabular}{lc}
\hline \multicolumn{1}{c}{ Tratamiento } & Nomenclatura \\
\hline $50 \mathrm{ml}$ de extracto acuoso & EA1 \\
$100 \mathrm{ml}$ de extracto acuoso & EA2 \\
$150 \mathrm{ml}$ de extracto acuoso & EA3 \\
$50 \mathrm{ml}$ de extracto hidroalcohólico & EH1 \\
$100 \mathrm{ml} \mathrm{de} \mathrm{extracto} \mathrm{hidroalcohólico}$ & EH2 \\
$150 \mathrm{ml}$ de extracto hidroalcohólico & EH3 \\
\hline
\end{tabular}

El diseño fue de completamente al azar con tres réplicas por tratamiento más tres réplicas de la muestra control. La distribución espacial de los tratamientos se observa en la Figura 1. 


\begin{tabular}{|c|c|c|c|c|c|c|}
\hline EH50 & EA50 & EA150 & MC & EH50 & EH150 & EH100 \\
\hline MC & EA100 & EA50 & EA150 & EH100 & MC & EH150 \\
\hline EA100 & EA150 & EA100 & EA50 & EH150 & EH50 & EH100 \\
\hline
\end{tabular}

Figura 1. Distribución espacial de las unidades experimentales

Las unidades experimentales se mantuvieron en condiciones controladas de laboratorio con temperatura entre 25 a $27^{\circ} \mathrm{C}$ y se mezclaron cada siete días para mantener las condiciones aerobias. De cada unidad experimental se tomaron muestras cada 15 días para medir el HEM. La monitorización de la contaminación de las muestras basado en el HEM se realizó por un lapso de 45 días, contados desde el momento de la aplicación de los tratamientos. Además, se midió el $\mathrm{pH}$ al inicio y al final del proceso de biorremediación.

El porcentaje de remediación de HEM se determinó mediante la ecuación 1.

$$
\% D i s=\frac{H E M_{i}-H E M_{f}}{H E M_{i}} \times 100
$$

Dónde:

$H E M_{i}=$ Extraíbles con hexano inicial, \%

$H E M_{f}=$ Extraíbles con hexano final, $\%$

$\%$ Dis $=$ Porcentaje de remediación

\section{Tratamiento estadístico}

Se aplicó un análisis ANDEVA multifactorial con los factores experimentales: Tipo de Extracto, Cantidad de Extracto Aplicado y Tiempo de tratamiento y la variable respuesta fue el HEM medido. Adicionalmente, se realizó un análisis de múltiples rangos utilizando la prueba Tukey HSD para establecer diferencias significativas entre los valores de HEM medidos respecto a los factores que influyeron significativamente. La misma prueba se utilizó para establecer si los tratamientos muestran diferencias respecto a la muestra control. Los análisis se realizaron en el software estadístico Statgraphics XVII.

\section{RESULTADOS}

El resultado de la textura de la muestra de suelo utilizada se manifiesta con una textura franco arenosa, con mayoría de partículas de arena (Tabla 4). Esta propiedad no se vio influenciada por la contaminación con gasoil, ya que la misma se determina a partir de la fracción mineral del suelo. Por lo tanto, es independiente de otros componentes que puedan estar presentes (agua, aceite, materia orgánica).

Tabla 4

Resultados de la textura del suelo

\begin{tabular}{lcccc}
\hline \multirow{2}{*}{ Muestra } & \multicolumn{4}{c}{ Textura } \\
\cline { 2 - 5 } \multicolumn{1}{c}{ A } & Ar & L & Tipo \\
\hline Criginal & 68.0 & 13.2 & 18.8 & Fa \\
Contaminada & 67.6 & 13.5 & 18.9 & Fa \\
\hline
\end{tabular}


El pH del suelo también se mantuvo constante a pesar de la contaminación con gasoil (Tabla 5), lo que demuestra que la presencia de los hidrocarburos en el suelo no afectó el intercambio de electrones que genera el potencial eléctrico medido por el potenciómetro en unidades de $\mathrm{pH}$.

Tabla 5

Propiedades fisicoquímicas del suelo

\begin{tabular}{lllllll}
\hline Muestra & H & DR & pH & MO & CO & HEM \\
\hline Original & 0.7 & 2.6 & 4.3 & 2.8 & 1.6 & 0.4 \\
Contaminada & 1.1 & 1.5 & 4.3 & 3.9 & 2.4 & 8.2 \\
\hline
\end{tabular}

El suelo presenta características que lo clasifican como fuertemente ácido, según la Guía para la Evaluación de la Calidad y Salud del Suelo (USDA, 1999). Las demás propiedades del suelo se vieron afectadas por la presencia de gasoil; en el caso de la densidad real, se observa como en el suelo contaminado, esta disminuye, lo que se debe a que al ser el gasoil menos denso que el suelo, al ocupar los espacios porosos, provoca la disminución de esta propiedad.

Las demás propiedades medidas aumentaron en el suelo contaminado, siendo el porcentaje de extraíbles con hexano la más afectada, debido a que el hexano extrae los hidrocarburos adicionados al suelo, por ser estos solubles en esta sustancia química, lo que produce un aumento en este parámetro medido.

En todas las unidades experimentales el porcentaje de aceites y grasas disminuyó respecto al tiempo, lo que era de esperarse, incluso en la muestra control a la que no se le aplicó tratamiento alguno. La disminución del porcentaje de HEM en las unidades experimentales fue mayor que en la unidad control (MC), siendo el tratamiento más eficiente el EH150 con un 93.90\% de disminución (Tabla 6).

Tabla 6

Resultado del proceso de remediación del suelo contaminado con gasoil

\begin{tabular}{cccccccc}
\hline Días & MC & EA1 & EA2 & EA3 & EH1 & EH2 & EH3 \\
\hline 0 & 8.2 & 8.2 & 8.2 & 8.2 & 8.2 & 8.2 & 8.2 \\
15 & 3.9 & 3.7 & 2.2 & 1.9 & 3.6 & 2.4 & 1.5 \\
30 & 3.6 & 2.6 & 1.7 & 1.3 & 2.4 & 1.8 & 1.1 \\
45 & 3.2 & 1.7 & 1.2 & 1.1 & 1.9 & 1.2 & 0.5 \\
\hline \%Dis & 60.9 & 79.3 & 85.4 & 86.6 & 76.8 & 85.4 & 93.9 \\
\hline
\end{tabular}

Se demuestra a través de los resultados que los tratamientos con $S$. saponaria fueron satisfactorios en el tiempo establecido de 45 días con porcentajes de remediación entre 76.8 y $93.9 \%$, superando a la atenuación natural que logró un $60.9 \%$ (Tabla 6).

Se proyecta que las unidades experimentales lleguen a alcanzar el valor límite de 0.4 (HEM original del suelo) en menos de 90 días, con excepción de la muestra control (Figuras 2 y 3). 


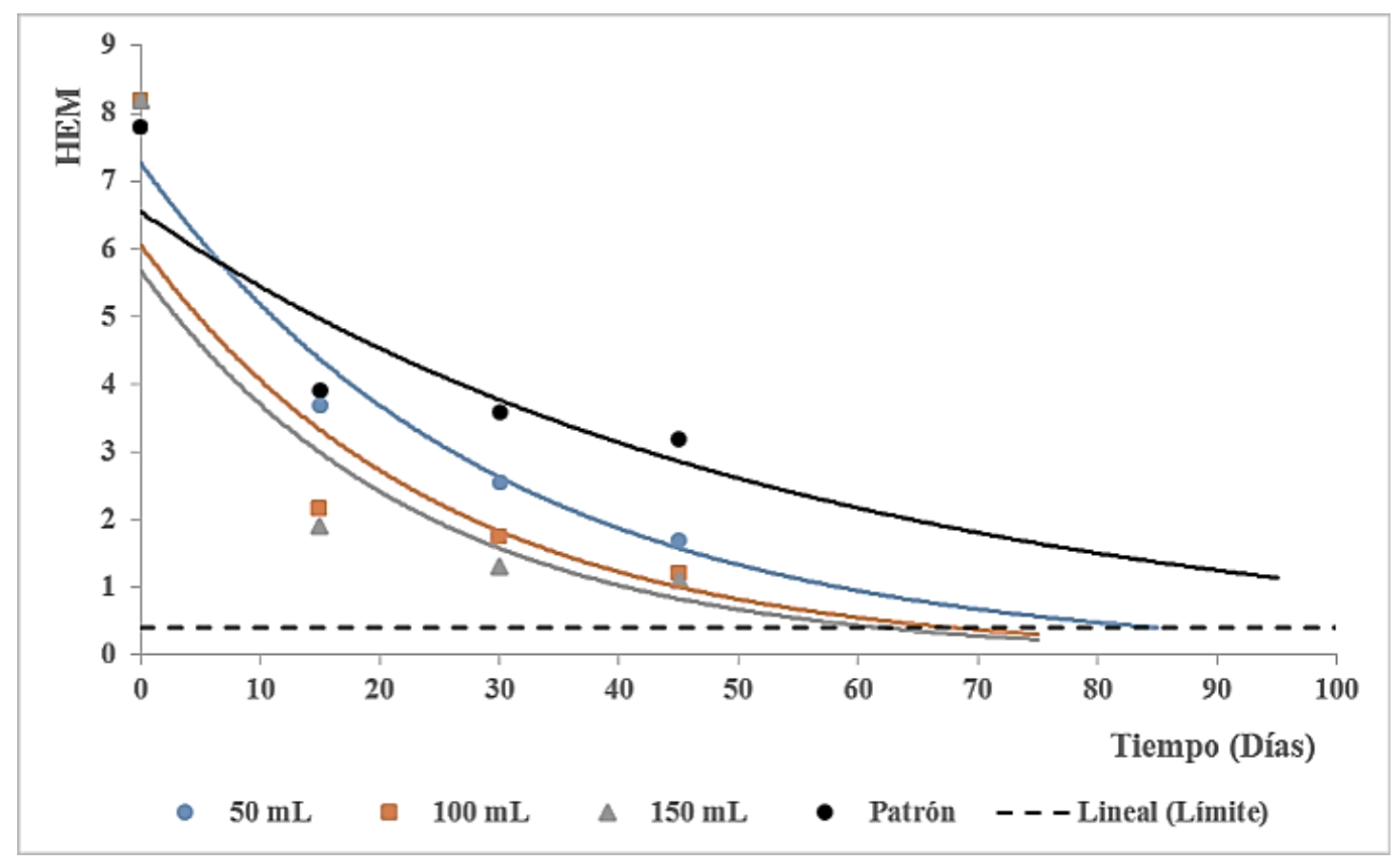

Figura 2. Representación del proceso de remediación con extracto acuoso

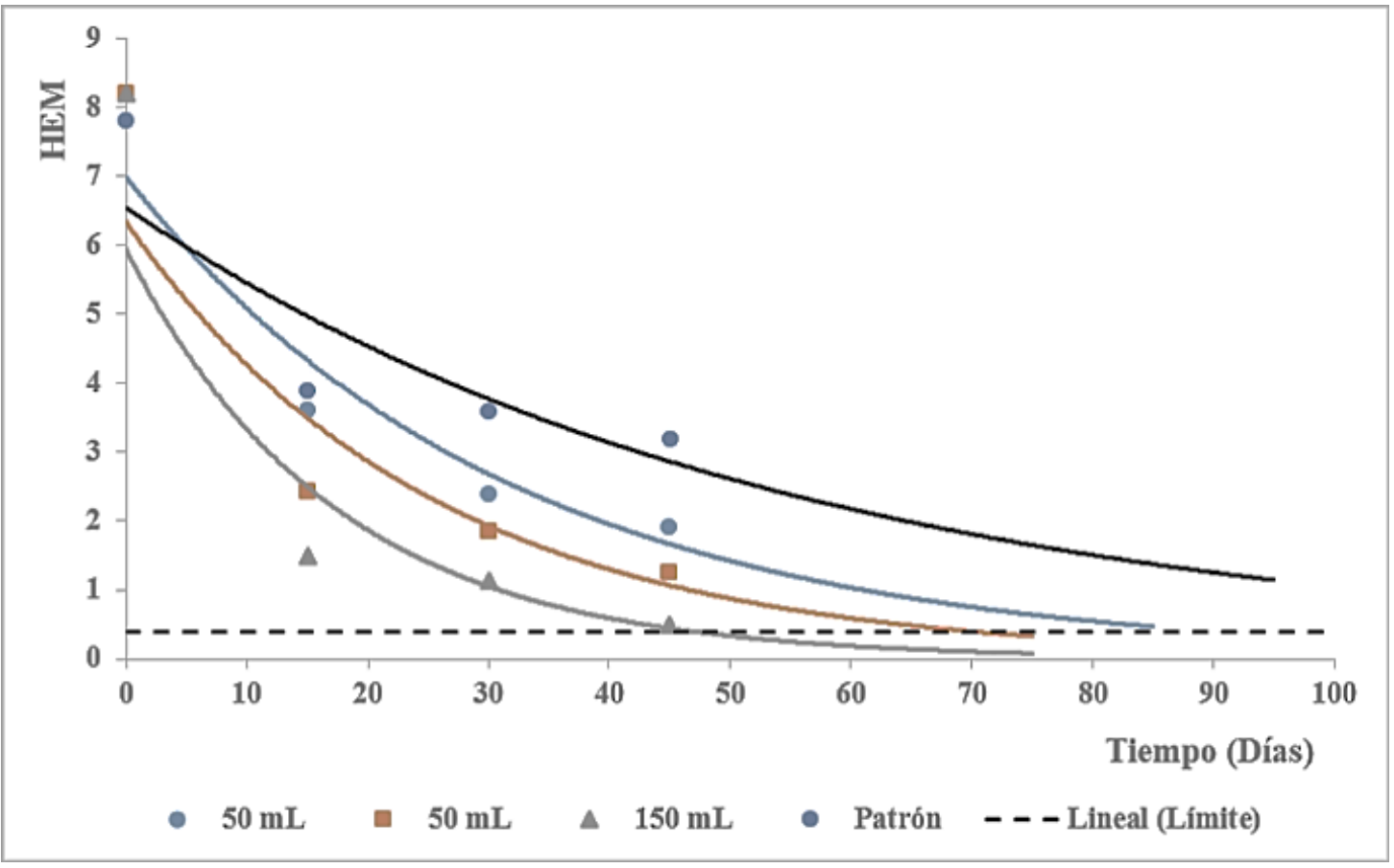

Figura 3. Representación del proceso de remediación con extracto hidroalcohólico

El pH varía poco durante todo el proceso, siendo constante para tratamientos como EA2, EH3 y MC. Se observó un ligero aumento en EH1 y EH2 y disminuyó en EA1 y EA3 (Tabla 7). 
Tabla 7

Variación de pH durante el proceso de remediación

\begin{tabular}{cccccccc}
\hline Días & MC & EA1 & EA2 & EA3 & EH1 & EH2 & EH3 \\
\hline 0 & 4.3 & 4.3 & 4.3 & 4.3 & 4.3 & 4.3 & 4.3 \\
45 & 4.3 & 4.2 & 4.3 & 4.1 & 4.4 & 4.4 & 4.3 \\
\hline
\end{tabular}

Tanto la cantidad de extracto y tiempo tuvieron influencia estadísticamente significativa sobre la concentración de HEM (p < 0.05) (Tabla 8). Sin embargo, el tipo de extracto (Acuoso o Hidroalcohólico) no influyó en los resultados de HEM medidos. Por lo tanto, se puede usar cualquiera de los dos con resultados estadísticamente similares. En relación a las interacciones, se observa que solo la combinación entre la Cantidad de extracto y el Tiempo influyeron en los valores de HEM $(\mathrm{p}<0.05)$.

\section{Tabla 8}

Análisis de varianza multifactorial para HEM

\begin{tabular}{lccccc}
\hline \multicolumn{1}{c}{ Fuente } & Suma de Cuadrados & gl & Cuadrado Medio & F & Valor-P \\
\hline A:Tipo de Extracto & 0.03227 & 1 & 0.03227 & 1.22 & 0.3124 \\
B:Cantidad de extracto (ml) & 4.52116 & 2 & 2.26058 & 85.19 & 0.0000 \\
C:Tiempo (Días) & 162.892 & 3 & 54.2974 & 2046.3 & 0.0000 \\
INTERACCIONES & & & & & \\
AB & 0.17716 & 2 & 0.08858 & 3.34 & 0.1060 \\
AC & 0.01177 & 3 & 0.00392 & 0.15 & 0.9274 \\
BC & 2.06767 & 6 & 0.34461 & 12.99 & 0.0033 \\
RESIDUOS & 0.15921 & 6 & 0.02654 & & \\
TOTAL (CORREGIDO) & 169.861 & 23 & & & \\
\hline
\end{tabular}

Al aplicar el análisis de rangos múltiples de Tukey al factor cantidad de extracto, se obtuvieron los resultados que se muestran en la Tabla 9. Se observa que existe diferencia significativa entre los resultados obtenidos para cada Cantidad de extracto aplicada, de acuerdo a la alineación de los X, en distintas posiciones dentro de los grupos homogéneos, lo que indica que los resultados son dependientes de este factor y que su valor influye de manera significativa sobre la variable HEM en el suelo contaminado.

Tabla 9

Pruebas de múltiples rangos para HEMporcantidad de extracto $(\mathrm{ml})$

\begin{tabular}{ccccccc}
\hline Cantidad de extracto (ml) & Casos & Media LS & Sigma LS & Grupos Homogéneos \\
\hline 150 & 8 & 2.89 & 0.058 & $\mathrm{X}$ & & \\
100 & 8 & 3.28 & 0.058 & & $\mathrm{X}$ & \\
50 & 8 & 3.94 & 0.058 & & & $\mathrm{X}$ \\
\hline
\end{tabular}

Existen diferencias significativas en los valores de HEM en cada uno de los días donde se realizó el muestreo y análisis (Tabla 10). Como era de esperarse, donde se reportó el menor valor de HEM fue a los 45 días. Lo anterior demuestra que los hidrocarburos presentes en el suelo se van degradando por efecto de la acción microbiana conforme van pasando los días. 
Tabla 10

Pruebas de múltiples rangos para HEMpor tiempo (dias)

\begin{tabular}{ccccccc}
\hline Tiempo (Días) & Casos & Media LS & Sigma LS & Grupos Homogéneos \\
\hline 45 & 6 & 1.28 & 0.067 & $\mathrm{X}$ & & \\
30 & 6 & 1.83 & 0.067 & & $\mathrm{X}$ & \\
15 & 6 & 2.56 & 0.067 & & & $\mathrm{X}$ \\
0 & 6 & 8.20 & 0.067 & & & \\
\hline
\end{tabular}

Para establecer si los resultados obtenidos al aplicar los extractos fueron diferentes a los medidos en la muestra patrón se realizó ANDEVA simple, por cada tipo de extracto de forma individual.

Al comparar los resultados obtenidos con el Extracto Acuoso y la Muestra Control mediante ANDEVA se observó que existe diferencia significativa $(\mathrm{F}=7.26 ; \mathrm{p}=0.0113)$. En la Tabla 11 se muestra el resultado de la prueba de contraste de Dunnet asociada a la prueba de varianza.

\section{Tabla 11}

Prueba de múltiples rangos de Dunnet para la comparación entre la eficiencia del Extracto Acuoso y la Muestra Control

\begin{tabular}{ccccc}
\hline Tratamiento & Casos & Media LS & Grupos homogéneos \\
\hline EA3 & 3 & 1.44667 & $\mathrm{X}$ & \\
EA2 & 3 & 1.70333 & $\mathrm{X}$ & $\mathrm{X}$ \\
EA1 & 3 & 2.65333 & $\mathrm{X}$ & $\mathrm{X}$ \\
MC & 3 & 3.58667 & & \\
\hline
\end{tabular}

Se observa que el tratamiento EA1 no presentó diferencia significativa respecto a la MC, como sí lo mostraron los tratamientos EA2 y EA3. Lo que indica que al aplicar $50 \mathrm{ml}$ de extracto acuoso no se logra ningún efecto significativo comparado en la atenuación natural.

Al comparar los resultados obtenidos con el Extracto Hidroalcohólico y la Muestra Control mediante ANDEVA se observó que existe diferencia significativa $(F=9.50 ; p=0.0052)$. En la Tabla 12 se muestra el resultado de la prueba de contraste de Dunnet asociada a la prueba de varianza

\section{Tabla 12}

Prueba de múltiples rangos de Dunnet para la comparación entre la eficiencia del Extracto Hidroalcohólico y la Muestra Control

\begin{tabular}{cccccc}
\hline Tratamiento & Casos & Media LS & Grupos Homogéneos \\
\hline EH3 & 3 & 1.03667 & $\mathrm{X}$ & & \\
EH2 & 3 & 1.83667 & $\mathrm{X}$ & $\mathrm{X}$ & \\
EH1 & 3 & 2.63667 & & $\mathrm{X}$ & $\mathrm{X}$ \\
MC & 3 & 3.58667 & & & $\mathrm{X}$ \\
\hline
\end{tabular}

Se observa que las EH2 y EH3 mostraron diferencias significativas respecto a MC y al igual que se observó en EA, el tratamiento con $50 \mathrm{ml}$ de extracto (EH1) no mostró diferencia significativa respecto al MC. 


\section{DISCUSIÓN}

Respecto a las características del suelo, el mismo posee baja humedad, aun cuando al contaminarse esta propiedad aumenta. La cantidad de materia orgánica puede considerarse como alta (Cáceres, 2013) y se observa que el gasoil produce un aumento importante en este parámetro, así como del carbono orgánico, lo que se debe a la presencia de los hidrocarburos que aportan carbono; sin embargo, esto es desfavorable, ya que este carbono no puede ser utilizado por las plantas debido a su origen mineral. Asimismo, se observó que la incorporación de los extractos de $S$. saponaria no causó ningún efecto o variación importante en la condición de acidez del suelo, manteniéndose al final del proceso como un suelo fuertemente ácido (USDA, 1999).

Durante el proceso de remediación, se observó que en todas las unidades experimentales se presentó una disminución del contenido de hidrocarburos extraíbles con hexano, incluso en la unidad experimental donde no se aplicó tratamiento, lo que indica que el suelo fue capaz de remediarse por atenuación natural, sobre todo por tratarse de gasoil, cuyos componentes al estar en el suelo están sujetos a fotooxidación y degradación por parte de los microorganismos propios del suelo (Repsol, 2016). Las diferencias entre técnicas de remediación y atenuación natural son también corroboradas al estudiar la combinación de fitorremediación y bioaumentación cuya eficiencia fue de $68 \%$, superior a la atenuación natural que reportó 37\% (Agnello et al., 2016), resultados menores a los obtenidos en la presente investigación (93.9\% por biorremediación y $60.9 \%$ por atenuación natural). Lo anterior también fue observado por Arrieta et al. (2012), quienes reportan para un suelo contaminado con gasoil un porcentaje de remediación de $36.86 \%$ por atenuación natural, valor menor al observado en la presente investigación, el cual fue de $60.98 \%$. Otra investigación que estudió técnicas de biorremediación en comparación con la atenuación natural muestra resultados menores a los obtenidos en la presente investigación y reporta una eficiencia de remediación por Landfarming asistido con bioaumentación de $89 \%$ y $57 \%$ por atenuación natural (Guarino et al., 2017). Las diferencias en los resultados pueden deberse a la procedencia de los suelos y a que presentaron diferentes propiedades fisicoquímicas (Martínez-Prado et al., 2011; Ferreira et al., 2013). Asimismo, la diferencia de especies de microorganismos presentes en el suelo también es un factor fundamental para el proceso de biorremediación (Aparicio et al., 2019). Resultados similares fueron obtenidos por Romaniuk et al. (2007), quienes reportan que en un suelo franco contaminado con gasoil y aceite de cárter se observa atenuación natural; sin embargo, de forma más lenta que al agregarle un producto comercial.

La eficiencia mayor de remediación obtenida al agregar los extractos de S. saponaria se puede deber no solo a que proporciona materia orgánica que sirve como nutrimento a los microorganismos y a la presencia de surfactantes naturales; sino también al contenido de agua que adiciona el suelo, ya que se ha reportado que el porcentaje de disminución de los hidrocarburos contaminantes en un suelo arenoso es proporcional a la cantidad de agua agregada al mismo en el proceso de remediación (Corona-Ramírez \& Iturbe-Argüelles, 2005).

El contenido de sustancias surfactantes en los extractos (saponina) puede contribuir a crear emulsiones entre los hidrocarburos y el agua, lo que puede a su vez hacerlos más biodisponibles para los microorganismos. La actividad de las saponinas como surfactantes fue reportada en investigaciones donde trabajaron con saponinas extraídas de Chenopodium quinoa Willd (Ahumada et al., 2016). Por otro lado, se lograron producir nonoemulsiones utilizando saponinas de quillay, lo que es indicativo de que estos compuestos presentes en los extractos de $S$. saponaria pueden ser precursores de emulsiones de hidrocarburos en agua (Riquelme \& Arancibia, 2019). También se demostró que extractos acuosos vegetales, como el de la fibra de Cocos nucifera, son efectivos para acelerar el proceso de remediación de un suelo contaminado con hidrocarburos (Marín et al., 2018), con el que se logró un máximo de eficiencia de $90.14 \%$, valor mayor al $86.59 \%$, máximo obtenido para el Extracto Acuoso de $S$. saponaria en la presente investigación. Sin embargo, se debe tomar en cuenta que se utilizaron diferentes contaminantes y los extractos también fueron diferentes, por lo que es de esperarse resultados que difieren.

Otro factor que influyó de manera importante en el efecto ejercido por el extracto de $S$. saponaria fue la presencia del alcohol en el extracto, efecto positivo que también fue observado al utilizar un producto comercial a base de alcohol para la remediación de un suelo contaminado con aceite de motor (Moya- 
Ramírez et al., 2014). De igual forma, se destaca el estudio del uso del alcohol etílico como extractor de sustancia húmicas, por lo que está clara su influencia sobre la materia orgánica en el suelo y que el mismo puede actuar, además sobre los hidrocarburos presentes en el suelo contaminado (García-Díaz et al., 2015). También se puede mencionar que alcoholes como el etílico y el isopropílico pueden acelerar el proceso de atenuación natural de un suelo contaminado con gasoil (Pérez et al., 2015), lo que es consistente con el resultado de la presente investigación. Del mismo modo, se reporta que el extracto hidroalcohólico de cáscaras de naranja acelera el proceso de biorremediación en un suelo franco arenoso arcilloso contaminado con un petróleo crudo liviano, con un máximo de eficiencia de 90.9\% en 42 días (Marín, 2017), resultado que fue menor al máximo obtenido en la presente investigación de $93.90 \%$ en 45 días. Aunque se puede decir que son coincidentes pues ambos extractos actúan de manera similar. Por otra parte, el estudio del efecto de un extracto hidroalcohólico de cáscaras de naranja como remediador de una arena contaminada con gasoil reportó una eficiencia de $92.07 \%$ con una dosis de $100 \mathrm{ml}$ por kilogramo a un tiempo de 60 días (MarínVelásquez \& Barrutia-Barreto, 2020), resultado superior al observado para una dosis equivalente en el presente estudio, lo que indica que el tipo de extracto utilizado es determinante en el proceso de remediación. Asimismo, Marín \& Arriojas (2020) demostraron que el extracto hidroalcohólico de $C$. sinensis es capaz de remediar una arena contaminada con gasolina hasta un máximo de $96.42 \%$, un porcentaje mayor al obtenido con los extractos estudiados en la presente investigación; sin embargo, es claro que el uso de hidrocarburos más livianos genera una mayor remoción de los mismos.

El análisis ANDEVA factorial mostró que no existe diferencia significativa en la eficiencia de ambos extractos (Acuoso e Hidroalcohólico) por lo que no se presentan diferencias al aplicarlos bajo las condiciones de la investigación. Los factores que influyeron fueron la cantidad de extracto aplicada y el tiempo de exposición al producto. Estos mismos factores fueron estudiados al utilizar el extracto de cáscaras de naranja sobre un suelo contaminado con petróleo crudo y se obtuvieron resultados similares (Marín, 2017). Es evidente que la aplicación de una mayor cantidad de extracto de $S$. saponaria al suelo contaminado aumenta la efectividad de la biorremediación, lo que se sustenta en la prueba de múltiples rangos. La influencia de la cantidad de extracto y del tiempo obtenido coincide con lo obtenido por Marín et al. (2018), quienes demostraron que estos factores también influyeron en la remediación de un suelo contaminado con petróleo al tratarse con extracto de fibra de coco. Los resultados demostraron que al aplicar los extractos de $S$. saponaria, el proceso de remediación mostró diferencias estadísticamente significativas respecto a la muestra control, lo que corrobora igualmente la eficiencia de los tratamientos aplicados al ser comparados con la atenuación natural. La eficiencia de la bioestimulación también fue estudiada para remediar un suelo agrícola contaminado con diésel, donde se concluye que la bioestimulación es la alternativa más factible para la remoción de los contaminantes del suelo, en comparación con la atenuación natural (Islas-García et al., 2016). También se reportaron resultados concordantes con los obtenidos en la presente investigación, al bioestimular un suelo contaminado con hidrocarburos aplicando bagazo y cachaza de caña, con diferencias significativas respecto a la atenuación natural (Rodríguez, 2017).

\section{CONCLUSIONES}

Los resultados del presente trabajo mostraron que la aplicación de extractos de S. saponaria (Acuoso e Hidroalcohólico) logra aumentar la eficiencia de remoción de hidrocarburos de gasoil, que contaminan un suelo. Se concluye que para el suelo estudiado y bajo las condiciones experimentales, la aplicación de los extractos naturales de $S$. saponaria es un tratamiento efectivo para la remediación.

Los resultados de la investigación se pueden considerar como básicos para continuar investigando sobre el uso de los extractos de $S$. saponaria como remediador de suelos contaminados con hidrocarburos, ya que se obtuvieron resultados satisfactorios. Sin embargo, se debe continuar investigando con otras metodologías de análisis que permitan establecer el mecanismo de acción, así como el efecto sobre hidrocarburos específicos como los aromáticos. 


\section{REFERENCIAS}

Agnello, A. C., Bagard, M., van Hullebusch, E. D., Esposito, G., \& Huguenot, D. (2016). Comparative bioremediation of heavy metals and petroleum hydrocarbons co-contaminated soil by natural attenuation, phytoremediation, bioaugmentation and bioaugmentation-assisted phytoremediation. Science of the Total Environment, 564, 693-703. https://doi.org/10.1016/j.scitotenv.2015.10.061

Ahumada, A., Ortega, A., Chito, D., \& Benítez, R. (2016). Saponinas de quinua (Chenopodium quinoa Willd.): un subproducto con alto potencial biológico. Revista Colombiana de Ciencias Químicas y Farmacéuticas, 45(3), 438-469.http://dx.doi.org/10.15446/rcciquifa.v45n3.62043

Alarcón, K. L. (2016). Extracción de saponinas del fruto de la Sapindus saponaria (choloque), y sus aplicaciones. Revista Ingeniería: Ciencia, Tecnología e Innovación, 3(1), 37-41. http://revistas.uss.edu.pe/index.php/ING/article/view/356

Aparicio, J. D., García-Velasco, N., Urionabarrenetxea, E., Soto, M., Álvarez, A., \& Polti, M. A. (2019). Evaluation of the effectiveness of a bioremediation process in experimental soils polluted with chromium and lindane. Ecotoxicology and Environmental Safety, 181, 255-263. https://doi.org/10.1016/j.ecoenv.2019.06.019

Arrieta, O. M., Rivera, A. P., Arias, L., Rojano, B. A., Ruiz, O., \& Cardona, S. A. (2012). Biorremediación de un suelo con diésel mediante el uso de microorganismos autóctonos. Gestión y Ambiente, 15(1), $27-$ 39. https://www.redalyc.org/pdf/1694/169424101004.pdf

Bazán, R. (2017). Manual de procedimientos de los análisis de suelos y agua con fines de riego. Ministerio de Agricultura y Riego, Perú. https://tinyurl.com/ycoyjbbm

Benavides, J., Quintero, G., Guevara, A. L., Jaimes, D. C., Gutiérrez, S. M., \& Miranda, J. (2006). Bioremediación de suelos contaminados con hidrocarburos derivados del petróleo. Nova Publicación Cientifica, 4(5), 82-90. https://www.redalyc.org/pdf/411/41140509.pdf

BP Oil España. (2000). Datos sobre higiene, seguridad y medio ambiente de productos BP. Gasóleo. https://tinyurl.com/yx9vwxpf

Cáceres, F. L. (2013). Determinación de Materia Orgánica en Suelos y Sedimentos. https://tinyurl.com/yxjq9v2p

Chan-Quijano, J. A., Jarquín-Sánchez, A., Ochoa-Gaona, S., \& Martínez-Zurimendi, P. (2015). Directrices para la remediación de suelos contaminados con hidrocarburos. Teoría y Praxis, 17, 123-144. https://www.redalyc.org/pdf/4561/456144902006.pdf

Corona-Ramírez, L., \& Iturbe-Argüelles, R. (2005). Atenuación natural en suelos contaminados con hidrocarburos. Ingeniería. Investigación y Tecnología, 2, $119-126$. https://www.redalyc.org/pdf/404/40460203.pdf

Díaz-Martínez, M. E., Alarcón, A., Ferrera-Cerrato, R., Almaraz-Suarez, J. J., \& García-Barradas, O. (2013). Crecimiento de Casuarina equisetifolia (Casuarinaceae) en suelo con diésel, y aplicación de bioestimulación y bioaumentación, Revista de Biología Tropical, 61(3), 1039-1052. https://www.scielo.sa.cr/pdf/rbt/v61n3/a05v61n3.pdf

Ferreira, T. C., Santos, F. J., \& Pessoa, F. (2013). Biorremediación de un suelo tropical contaminado con residuos aceitosos intemperizados. Revista Internacional de Contaminación Ambiental, 29(1), 21 28. http://www.scielo.org.mx/pdf/rica/v29n1/v29n1a2.pdf

García-Díaz, C., Nebbioso, A., Piccolo, A., Barrera-Cortés, J., \& Martínez-Palou, R. (2015). Remediation of Hydrocarbon-Contaminated Soil by Washing with Novel Chemically Modified Humic Substances. Journal of Environmental Quality, 44(6), 1764-1771. https://doi.org/10.2134/jeq2014.09.0399

Gómez, W., Gaviria, J., \& Cardona, S. (2009). Evaluación de la bioestimulación frente a la atenuación natural y la bioaumentación en un suelo contaminado con una mezcla de gasolina-diésel. Dyna, 76(160), 83-93. https://www.redalyc.org/pdf/496/49612068032.pdf

Guarino, C., Spada, V., \& Sciarrillo, R. (2017). Assessment of three approachesof bioremediation (Natural Attenuation, Landfarming and Bioagumentation - Assistited Landfarming) for a petroleum hydrocarbons contaminated soil. Chemosphere, $170,10-16$. https://doi.org/10.1016/j.chemosphere.2016.11.165 
Islas-García, A., Peralta-Rodríguez, M., Vega-Loyo, L., López-Aguilar, R., \& Rodríguez-Vázquez, R. (2016). Biorremediación por bioestimulación y bioaumentación con microorganismos nativos de un suelo a gricola contaminadoron hidrocarburos. https://www.researchgate.net/publication/328389954

Laczi, K., Kis, A., Horváth, B., Maróti, G., Hegedüs, B., Perei, K., \& Rákhely, G. (2015). Metabolic responses of Rhodococcus erythropolis PR4 grown on diesel oil and various hydrocarbons. Applied Microbiology and Biotechnology, 99(22), 9745-9759.https://doi.org/10.1007/s00253-015-6936-z

Lizcano, R., Olivera, D., Saavedra, D., Machado, L., Valencia, E. R., Moreno, M. F., \& Flores, M. F. (2017). Muestreo de suelos, técnicas de laboratorio e interpretación de análisis de suelos. Centro de Formación Agroindustrial La Angostura. https://www.researchgate.net/publication/323823646

Loroña, F., Gómez, W., Joco, F., Reynaga, C., Guiño, M., Gamarra, J., Díaz, F., Huamán, N., Gutiérrez, P., Mayte, J., Morán, M., \& Carhuancho, M. (2018). Eficiencia de la biorremediación de suelos contaminados con Diesel B5 mediante Microorganismo Eficaces (EM). Cátedra Villarreal, 6(2), 189-209. http://dx.doi.org/10.24039/cv201862278

Marín, T. (2017). Extracto hidroalcohólico de cáscaras de naranja (Citrus sinensis) como bioestimulador en un suelo de sabana contaminado con petróleo, Ingeniería, 21(2), 1-10. https://www.redalyc.org/pdf/467/46753192001.pdf

Marín, T. D., Gota, C. N., \& Ortiz, T. C. (2018). Evaluación del extracto obtenido como lixiviado de fibra de coco (Cocos nucifera) como bioestimulante en la remediación de un suelo contaminado con petróleo. Enfoque UTE, 9(4), 180-193. https://doi.org/10.29019/enfoqueute.v9n4.303

Marín, T. D., \& Arriojas, D. D. J. (2020). Efecto del extracto hidroalcohólico de cáscaras de naranja dulce (Citrus sinensis) en la remediación de una arena contaminado con gasolina. Geominas, 48(82), 6168. https://www.researchgate.net/publication/343638966

Marín-Velásquez, T., \& Barrutia-Barreto, I. (2020). Extracto hidroalcohólico de cáscara de Citrus sinensis y aceite de Pinus patula, una alternativa de biorremediación. Journal of Selva Andina Research Society, 11(2), 103-113. https://doi.org/10.36610/j.jsars.2020.110200103

Martínez-Prado, A., Pérez-López, M.A., Pinto-Espinoza, J., Gurrola-Narváez, B.A., \& Osorio-Rodríguez, A.L. (2011). Biorremediación de suelo contaminado con hidrocarburos empleando lodos residuales como fuente alterna de nutrientes. Revista Internacional de Contaminación Ambiental, 27(3), 241252. http://www.scielo.org.mx/pdf/rica/v27n3/v27n3a9.pdf

Moya-Ramírez, I., García-Román, M., Henares-Jiménez, M., Jurado-Alameda, E., \& Altmajer-Vaz, D. (2014). Remediación de suelos contaminados con aceite de motor mediante tensioactivos altamente biodegradables. Avances en Ciencias e Ingeniería, 5(2), $21-29$. https://www.redalyc.org/articulo.oa?id=323631115002

Osoria, F. C. (2018). Proyecto de Investigación de Descontaminación de Suelos por Diesel en el Laboratorio de Construcción Eptisa (Tesis de maestría). Universidad de la Coruña, La Coruña, España. http://hdl.handle.net/2183/21512

Pérez, S. L., Silva, I. C., Peñuela, G. A., \& Cardona, S. A. (2015). Evaluación de biocombustibles e hidrocarburos del petróleo (gasolina y diésel) en un suelo: proceso de transporte y biorremediación. RevistaEIA, 12(2), 21-46. https://www.redalyc.org/pdf/1492/149240052004.pdf

Rajasekar, A. (2016). Biodegradation of Petroleum Hydrocarbon and Its Influence on Corrosion with Special Reference to Petroleum Industry. En K. Heimann O., Karthikeyan, S. Muthu S. (Eds.), Biodegradation and Bioconversion of Hydrocarbons. Environmental Footprints and Eco-design of Products and Processes (p. 307-336). Springer Link. https://doi.org/10.1007/978-981-10-0201-4_9

Repsol. (2016). Ficha de datos de seguridad. Diésel B5 (DB5 S-50). https://tinyurl.com/y6zbsg4t

Reglamento Técnico Centroamericano. (2006). Productos de petróleo. Aceite combustible diésel. Especificaciones. https://tinyurl.com/y6zawa8s

Riquelme, N., \& Arancibia, C. (2019). Obtención de nanoemulsiones utilizando saponinas de quillay como sustituto de un surfactante sintético. Mundo Nano, 12(23), 1-12. https://doi.org/10.22201/ceiich.24485691e.2019.23.67654 
Rodríguez, G. E. (2017). Remoción de hidrocarburos totales en suelos contaminados con petróleo mediante residuos de Cachaza y Bagazo de caña de azúcar. UCV - Scientia, 9(1), 59-66. http://revistas.ucv.edu.pe/index.php/UCV-SCIENTIA/article/view/1236

Romaniuk, R., Brandt, J. F., Rios, P. R., \& Giuffré, L. (2007). Atenuación natural y remediación inducida en suelos contaminados con hidrocarburos. Ciencias del Suelo (Argentina), 25(2), 139-149. https://tinyurl.com/y964k2rx

Sánchez, J. A., \& Silva, L. J. (2008). Estudio silvicultural de la especie Sapindus saponaria L. (jaboncillo) como base para su aprovechamiento silvoindustrial. Revista Colombia Forestal, 11, 71-81. http://www.scielo.org.co/pdf/cofo/v11n1/v11n1a05.pdf

Speigh, J. G. (2002). Handbook of Petroleum Product Analysis. Wiley \& Sons, Inc. https://tinyurl.com/y57s92ja

Trujillo, M. A., \& Ramírez, J. F. (2012). Biorremediación en suelos contaminados con hidrocarburos en Colombia. Revista de Investigación Agraria y Ambiental, 3(2), 37-48. https://doi.org/10.22490/21456453.952

USDA. (1999). Departamento de Agricultura de los Estados Unidos de Norteamérica. Guía para la Evaluación de la Calidady Salud del Suelo. https://tinyurl.com/y3hyohe4

Whaley, O., Orellana, A., Tenorio, M., Pérez, E., Mendosa, M., \& Pecho, O. (2009). Plantasy Vegetación de Ica. Un recurso para restauración y conservación. Darwin Iniciative. https://www.kew.org/science/tropamerica/icaperu/plantas_libro_lowres.pdf 